\title{
Eigenvalue Finite Difference Approximations for Regular and Singular Sturm-Liouville Problems
}

\author{
By Nabil R. Nassif
}

\begin{abstract}
This paper includes two parts. In the first part, general error estimates for "stable" eigenvalue approximations are obtained. These are practical in the sense that they are based on the discretization error of the difference formula over the eigenspace associated with the isolated eigenvalue under consideration. Verification of these general estimates are carried out on two difference schemes: that of Numerov to solve the Schrödinger singular equation and that of the central difference formula for regular Sturm-Liouville problems. In the second part, a sufficient condition for obtaining a "stable" difference scheme is derived. Such a condition (condition $(\mathrm{N})$ of Theorem 2.1) leads to a simple "by hand" verification, when one selects a difference scheme to compute eigenvalues of a differential operator. This condition is checked for one- and two-dimensional problems.
\end{abstract}

Introduction. In this work, we are concerned with eigenvalue-eigenvector approximation by finite difference methods for differential operators defined on functions with bounded or unbounded domains. Our results will be illustrated in particular for the Schrödinger radial operator whose "energy levels" are obtained numerically using difference schemes. Let

$$
L[y]=-y^{\prime \prime}+q(x) y, \quad 0<x<\infty,
$$

and consider the boundary conditions

$$
B[y]=c y^{\prime}(0)+d y(0)=0
$$

and

$$
y(x) \text { bounded on }(0, \infty) \text {. }
$$

Let $x_{i}=i h, 0 \leqslant i \leqslant N, x_{0}=0, X=x_{N}=N h$, with $\lim _{h \rightarrow 0} X=\lim _{h \rightarrow 0} N=\infty$.

Optimal error estimates for difference methods will depend on how $X(h)$ and $N(h)$ tend to $\infty$. For example (see Corollary 2.1), possible choices for $X(h)$ and $N(h)$ are, respectively, $m^{2}$ and $2^{m} m^{2}$, with $h=1 / 2^{m}$.

The Numerov [8] difference scheme consists in finding $Y=\left\{Y_{i}\right\}_{0 \leqslant i \leqslant N}, \lambda_{h} \in R$, such that

$$
\begin{gathered}
\left(-Y_{i-1}+2 Y_{i}-Y_{i+1}\right) / h^{2}+\left(q_{i-1} Y_{i-1}+10 q_{i} Y_{i}+q_{i+1} Y_{i+1}\right) / 12 \\
=\lambda_{h}\left(Y_{i-1}+10 Y_{i}+Y_{i+1}\right) / 12, \\
B_{h}[Y]=0,
\end{gathered}
$$

Received November 4, 1982; revised January 16, 1984 and October 8, 1986.

1980 Mathematics Subject Classification (1985 Revision). Primary 65L15: Seondary 34B25.

C1987 American Mathematical Society $0025-5718 / 87 \$ 1.00+\$ .25$ per page 
and

$$
Y_{N}=0
$$

$B_{h}$ is the difference approximation to $B$. The choice of $B_{h}$ should be such that its discretization error with respect to $B$ has the same order as that of $L_{h}$ with respect to $L$. When $c=0, d=1$, the choice of $B_{h}$ is obvious. When $c \neq 0$, and in the case of Numerov's scheme, one must extend the eigenfunction $y(x)$ on $(-2 h, 0)$, and use a difference approximation to $y^{\prime}(0)$ over the points $-2 h,-h, 0, h$, and $2 h$. It can be verified that, $Y_{N}, Y_{0}$, and $Y_{k}, k<0$, can be eliminated, and the system (1.4)-(1.6) is written in the form

$$
-\left(L_{h}[Y]\right)_{i}=\lambda_{h}\left[Y_{i}\right], \quad 1 \leqslant i \leqslant N-1,
$$

where $L_{h}: R^{N-1} \rightarrow R^{N-1}$.

It is the goal of this paper to present abstract results for the analysis of finite difference methods for eigenvalue problems. The results are sufficiently general, relatively simple, and easily applicable to specific difference methods, such as (1.7). We present stability and convergence estimates involving the "discretization error" of the difference formula over the eigenspace associated with the eigenvalue under consideration. Our results are similar to those obtained by Vainikko [13] for differential operators on bounded domains. The argument used is an adaptation of one introduced by Vainikko and used repeatedly by Osborn [10] for compact operators and by Descloux, Nassif and Rappaz [4] for Galerkin approximations to noncompact operators. It essentially reduces the analysis to that of an algebraic eigenvalue problem. Furthermore, our estimates are general in the sense that they can be applied to operators with functions of several variables. We should mention here results available in the literature. Our results should be compared to the approach of Stummel [12] which is based on the development of a very general framework for the analysis of a variety of approximation processes. It has been our aim to tailor our results to the analysis of difference methods. The results of Kreiss [7] are intimately related to the regularity of the solution, while Grigorieff's results [6] are concerned with compact operators.

The theorems of Part 1 depend directly on "stability conditions" (conditions A1 and A2). In Part 2, we present a general theory based on a condition to be satisfied by the discretization error of the difference formula (condition $(\mathrm{N})$ of Theorem 2.1) on the set $\left\{f \mid L f \in H_{h}\right\}$, where $H_{h}$ is a suitable finite element space.

In each part we have considered two applications: the Numerov scheme (1.4)-(1.6) for the Schrödinger equation and the three-point central difference scheme for regular Sturm-Liouville problems.

Two-dimensional problems can also be treated. The verification of condition (N) for the five-point difference scheme is sketched at the end of Part 2.

Part 1. Convergence Estimates for Isolated Eigenvalues of Finite Multiplicity.

1.1. Definition and Results. Let $U$ be a complex Banach space with norm $|\cdot|$ and $\left\{U_{h}\right\}_{h}$ a sequence of finite-dimensional spaces with norms $|\cdot|_{h}$. Consider also linear operators $L: U \rightarrow U$ with $D(L) \subset U, L_{h}: U_{h} \rightarrow U_{h}, r_{h}: U \rightarrow U_{h}$. For $u \in D(L)$, we define the discretization error associated with $u$ as

$$
e_{h}(u)=r_{h} L u-L_{h} r_{h} u \in U_{h} .
$$


Let $\sigma(L)$ be the spectrum of $L$ and let $\lambda \in \sigma(L)$ be an isolated eigenvalue of finite algebraic multiplicity $m$. Let $\Delta$ be a closed disk with center $\lambda$ and boundary $\Gamma$ such that $\Delta \cap \sigma(L)=\{\lambda\}$. Let $\lambda_{h, 1}, \ldots, \lambda_{h, m(h)}$ be eigenvalues of $L_{h}$, repeated according to their algebraic multiplicities and contained in $\Delta$. We assume that the sequence $\left\{L_{h}\right\}_{h}$ is such that

(A1) $\exists \varepsilon_{0}>0$, such that $\forall \varepsilon, 0<\varepsilon<\varepsilon_{0}, \exists h_{0}$ such that $\forall h, h<h_{0}$,

$$
\sigma\left(L_{h}\right) \cap\{z|| z-\lambda \mid<\varepsilon\}
$$

contains exactly $m$ eigenvalues (repeated according to their multiplicities) of $L_{h}$. Denote these by $\lambda_{h, 1}, \ldots, \lambda_{h, m}$.

For an operator $D, R_{z}(D)=(z-D)^{-1}$ denotes the resolvent operator. We also assume:

(A2) $\forall K$, compact sets $\subset \zeta(L)$, the resolvent set of $L, \exists h_{0}>0$, such that $\forall h<h_{0}, K \subset \zeta\left(L_{h}\right)$, the resolvent set of $L_{h}$; furthermore, $\exists c$ independent of $h$ such that $\left|R_{z}\left(L_{h}\right)\right|_{h} \leqslant c, \forall h<h_{0}, \forall z \in K$.

For $u_{h} \in U_{h}, X_{h}$ and $Y_{h}$ subspaces of $U_{h}$, let

$$
\begin{gathered}
\delta_{h}\left(u_{h}, Z_{h}\right)=\inf _{z_{h} \in Z_{h}}\left|u_{h}-z_{h}\right|_{h}, \\
\delta_{h}\left(Y_{h}, Z_{h}\right)=\sup _{\substack{\left.y_{h} \in Y_{h} \\
i \dot{y}_{h}\right|_{h}=1}}\left[\delta_{h}\left(y_{h}, Z_{h}\right)\right], \\
\hat{\delta}_{h}\left(Y_{h}, Z_{h}\right)=\max \left[\delta_{h}\left(Y_{h}, Z_{h}\right), \delta_{h}\left(Z_{h}, Y_{h}\right)\right] .
\end{gathered}
$$

$E=(2 \pi i)^{-1} \int_{\Gamma} R_{z}(L) d z$ is the spectral projector of $L$ relative to $\lambda$, and for $h$ small enough

$$
F_{h}=(2 \pi i)^{-1} \int_{\Gamma} R_{z}\left(L_{h}\right) d z
$$

is the spectral projector of $L_{h}$ relative to $\left\{\lambda_{h, i}\right\}, 1 \leqslant i \leqslant m . E(U)$ and $F_{h}\left(U_{h}\right)$ are, respectively, the $m$-dimensional invariant subspaces of $L$ and $L_{h}$ corresponding, respectively, to $\lambda$ and $\left\{\lambda_{h, i} \mid 1 \leqslant i \leqslant m\right\}$.

Finally, consider the mapping $r_{h}^{E}=\left.r_{h}\right|_{E(U)}: E(U) \rightarrow U_{h}$ and let $E_{h}=r_{h} E(U)$. We assume:

(A3) For $h$ small enough: $\operatorname{dim}\left(E_{h}\right)=m$;

furthermore, $r_{h}^{E}: E(U) \rightarrow E_{h}$ is a bijection with

$$
\left|r_{h}^{E}\right|_{U, U_{h}}=\sup _{\substack{u \in E(U) \\|u|=1}}\left|r_{h} u\right|_{h} \leqslant c_{1}, \quad\left|\left(r_{h}^{E}\right)^{-1}\right|_{U_{h}, U}=\sup _{\substack{u_{h} \in E_{h} \\\left|u_{h}\right|_{h}=1}}\left|\left(r_{h}^{E}\right)^{-1} u_{h}\right| \leqslant c_{2},
$$

with $c_{1}, c_{2}$ constants independent of $h$.

REMARK 1.1. Note that the assumption on $r_{h}$ is only local, i.e., uniform boundedness must be satisfied on the invariant subspace $E(U)$ only.

Finally, let us introduce the quantity

$$
\gamma_{h}=\sup _{\substack{u \in E(U) \\|u|=1}}\left|r_{h} L u-L_{h} r_{h} u\right|_{h}
$$

and assume

(A4) $\lim _{h \rightarrow 0} \gamma_{h}=0$.

We now state our results. 
THEOREM 1.1. There exists a constant $c$, independent of $h$, such that

$$
\delta_{h}\left(F_{h}\left(U_{h}\right), r_{h} E(U)\right) \leqslant c \gamma_{h} .
$$

Eigenvalue estimates are based on the following preliminary argument used by Osborn [10] in a different context.

Introduce the operator $\Lambda_{h}=\left.F_{h} r_{h}\right|_{E(U)}: E(U) \rightarrow F_{h}\left(U_{h}\right)$. We shall prove that $\Lambda_{h}$ is a bijection. Letting $\hat{L}=\left.L\right|_{E(U)}$ and $\hat{L}_{h}=\Lambda_{h}^{-1} L_{h} \Lambda_{h}$, one can see that these operators can be considered in $E(U)$, with $\hat{L}$ having the eigenvalue $\lambda$ of algebraic multiplicity $m$, and $\hat{L}_{h}$ the eigenvalues $\lambda_{h, 1}, \ldots, \lambda_{h, m}$.

THEOREM 1.2. Under the assumptions (A1)-(A3), there exists a constant $c$ independent of $h$ such that

$$
\left|\hat{L}-\hat{L}_{h}\right|_{E(U)} \leqslant c \gamma_{h}
$$

By the choice of basis in $E(U)$, Theorem 1.2 reduces our original task to a pure matrix problem.

Let $f$ be a holomorphic function defined in the neighborhood of $\lambda$. Writing $f(\hat{L})$, $f\left(\hat{L}_{h}\right)$ in terms of Dunford integrals, one verifies that

$$
\left|f(\hat{L})-f\left(\hat{L}_{h}\right)\right|_{E(U)} \leqslant c\left|\hat{L}-\hat{L}_{h}\right|_{E(U)}
$$

Using the classical properties of traces and determinants, one obtains Theorem 1.3a, b; Theorem 1.3c, $\mathrm{d}$ is a direct application of results quoted in Wilkinson [14, pp. 80-81]. Here, $\alpha$ is the ascent of the eigenvalue $\lambda$ of $L$ and $\beta$ the number of Jordan blocks of the canonical form of $\hat{L}$.

THEOREM 1.3. There exists a constant $c$ independent of $h$ such that for $h$ small enough,

(a) $\left|f(\lambda)-(1 / m) \sum_{i=1}^{m} f\left(\lambda_{h, i}\right)\right| \leqslant c \gamma_{h}$,

(b) $\left|f^{m}(\lambda)-\prod_{i=1}^{m} f\left(\lambda_{h, i}\right)\right| \leqslant c \gamma_{h}$,

(c) $\max _{1 \leqslant i \leqslant m}\left|\lambda-\lambda_{h, i}\right| \leqslant c\left(\gamma_{h}\right)^{1 / \alpha}$,

(d) $\min _{1 \leqslant i \leqslant m}\left|\lambda-\lambda_{h, i}\right| \leqslant c\left(\gamma_{h}\right)^{\beta / m}$.

1.2. Proofs. To obtain the above results, we need the following lemmas; throughout, $c$ is a generic constant. (A2) leads to

LEMMA 1.1. There exists $h_{0}>0$ such that $\left|F_{h}\right|_{h} \leqslant c, \forall h<h_{0}$.

Proof. One has for $u_{h} \in U_{h},\left|u_{h}\right|_{h}=1$,

$$
F_{h} u_{h}=\frac{1}{2 \pi i} \int_{\Gamma} R_{z}\left(L_{h}\right) u_{h} d_{z}
$$

and by using (A2), with $K$ replaced by $\Gamma$, one obtains,

$$
\left|F_{h}\right|_{h} \leqslant \frac{c}{2 \pi} \operatorname{meas}(\Gamma) \text {. }
$$

LEMMA 1.2. There holds

$$
\sup _{\substack{u \in E(U) \\|u|=1}}\left|r_{h} E_{h} u-F_{h} r_{h} u\right|_{h} \leqslant c \gamma_{h} .
$$


Proof. We write

$$
r_{h} E u-F_{h} r_{h} u=\frac{1}{2 \pi i} \int_{\Gamma}\left[r_{h} R_{z}(L)-R_{z}\left(L_{h}\right) r_{h}\right] u d z
$$

Now use the following identities,

$$
\begin{aligned}
z \in \Gamma: r_{h} R_{z}(L)-R_{z}\left(L_{h}\right) r_{h} & =R_{z}\left(L_{h}\right)\left[\left(z-L_{h}\right) r_{h}-r_{h}(z-L)\right] R_{z}(L) \\
& =R_{z}\left(L_{h}\right)\left[r_{h} L-L_{h} r_{h}\right] R_{z}(L),
\end{aligned}
$$

to get

$$
r_{h} E u-F_{h} r_{h} u=\int_{\Gamma}(2 \pi i)^{-1} R_{z}\left(L_{h}\right)\left[r_{h} L-L_{h} r_{h}\right] R_{z}(L) u d z .
$$

Clearly, since $R_{z}(L) u \in E(U)$, and using (A2) with $K$ replaced by $(\Gamma)$, this implies

$$
\left|r_{h} E u-F_{h} r_{h} u\right|_{h} \leqslant(1 / 2 \pi) \int_{\Gamma}\left|R_{z}\left(L_{h}\right)\right|_{h} c \gamma_{h}\left|R_{z}(L)\right||u| d z,
$$

which proves the result.

Using assumption (A3) and Lemma 1.2, one easily deduces

LEMMA 1.3. One has

$$
\delta_{h}\left(r_{h} E(U), F_{h}\left(U_{h}\right)\right) \leqslant c \gamma_{h} .
$$

We omit also the proof of the following elementary result.

LEMMa 1.4. Let $Y_{h}$ and $Z_{h}$ be two subspaces of $U_{h}$ with the same finite dimension. Let $P_{h}: Y_{h} \rightarrow Z_{h}$ be a linear operator such that

$$
\left|P_{h} y-y\right|_{h} \leqslant .5|y|_{h}, \quad y \in U_{h} .
$$

Then $P_{h}$ is a bijection and $\left|P_{h}^{-1} z\right| \leqslant 2|z|, z \in Z_{h}$. Furthermore,

$$
\sup _{\substack{z \in Z_{h} \\|z|_{h}=1}}\left|P_{h}^{-1} z-z\right|_{h} \leqslant 2 \sup _{\substack{y \in Y_{h} \\|y|_{h}=1}}\left|P_{h} y-y\right|_{h} .
$$

Proof of Theorem 1.1. Let $\theta_{h}=\left.F_{h}\right|_{E_{h}}: E_{h} \rightarrow F_{h}\left(U_{h}\right)$ (recall $E_{h}=r_{h} E(U)$ ); for $h$ small enough, $E_{h}$ and $F_{h}\left(U_{h}\right)$ have the same finite dimension $m$; on the other hand, (A4) implies $\lim _{h \rightarrow 0} \gamma_{h}=0$. Using Lemmas 1.2 and $1.4, \theta_{h}^{-1}$ exists for $h$ small enough and is such that

$$
\sup _{\substack{y \in F_{h}\left(U_{h}\right) \\\left|y_{h}\right|_{h}=1}}\left|\theta_{h}^{-1} y\right|_{h} \leqslant c .
$$

Furthermore,

$$
\sup _{\substack{y \in F_{h}\left(U_{h}\right) \\|.|_{h}=1}}\left|\theta_{h}^{-1} y-y\right|_{h} \leqslant c \gamma_{h},
$$

i.e., $\delta_{h}\left(F_{h}\left(U_{h}\right), r_{h} E(U)\right) \leqslant c \gamma_{h}$.

Proof of Theorem 1.2. Note that

$$
\hat{L}-\hat{L}_{h}=\hat{L}-\Lambda_{h}^{-1} L_{h} \Lambda_{h}=\Lambda_{h}^{-1} \Lambda_{h} \hat{L}-\Lambda_{h}^{-1} L_{h} \Lambda_{h},
$$

and for $u \in E(U)$, using $\Lambda_{h}=\theta_{h}=\theta_{h} r_{h}^{E}$ and $\Lambda_{h}^{-1}=\left(r_{h}^{E}\right)^{-1}\left(\theta_{h}\right)^{-1}$, we have for $u \in E(U)$ such that $|u|=1$,

$$
\hat{L} u=\hat{L}_{h} u=\Lambda_{h}^{-1}\left[\Lambda_{h} \hat{L} u-L_{h} \Lambda_{h} u\right] .
$$


From (A3) and (1.8) one sees that $\left|\hat{L} u-\hat{L}_{h} u\right| \leqslant c\left|\Lambda_{h} L u-L_{h} \Lambda_{h} u\right|_{h}$. Note that $L_{h} \Lambda_{h}=L_{h} F_{h} r_{h}=F_{h} L_{h} r_{h}$, so that $\Lambda_{h} L u-L_{h} \Lambda_{h} u=F_{h} r_{h} L u-F_{h} L_{h} r_{h} u$. Using the uniform boundedness of $F_{h}$ on $U_{h}$, we obtain immediately our result.

Remark 1.2. Note that the estimates depend solely on the discretization error of the difference scheme over the invariant subspace, i.e., $\gamma_{h}$.

Remark 1.3. In the selfadjoint case, since $\alpha=1$ and $\beta=m$, note from Theorem 1.3 (b) and (c) that every eigenvalue $\lambda_{h, i}$ converges to $\lambda$ with the same rate. This can be checked by a simple algebraic manipulation which we omit here (see [5]).

1.3. Application to Numerov's Scheme for the Schrödinger Operator. We let $U=L^{2}(0, \infty) ; U_{h}=R^{N-1}$; on $q(x)$ we make the following assumption:

(1) $q \in C^{\infty}(0, \infty) \cap C[0, \infty], q \rightarrow 0$ as $x \rightarrow \infty$,

(2) $\alpha=\inf _{x \in R_{+}} q(x)<0, \quad M=\sup _{x \in R_{+}}|q(x)|$,

(3) $q$ Lipschitz continuous on $(0, \infty)$.

In case of $c=0, d=1$ in (1.2), there exists an infinite sequence $\left\{\lambda_{k}\right\}_{k}$ of isolated eigenvalues of multiplicity 1 such that for all $k, \alpha<\lambda_{k}<0$, with $\lim _{k \rightarrow \infty} \lambda_{k}=0$. Furthermore, each corresponding eigenfunction has exactly $k-1$ positive zeros and tends exponentially to zero as $x \rightarrow \infty$. The eigenfunctions are in $C^{\infty}(0, \infty)$.

For $f \in U$, set $|f|=\left\{\int_{0}^{\infty}|f(x)|^{2} d x\right\}^{1 / 2}$, and for $f_{h} \in U_{h}, f_{h}=\left\{f_{h, i}\right\}_{i}$, set

$$
\left|f_{h}\right|_{h}=\left\{\sum_{i=1}^{N-1} h\left|f_{h, i}\right|^{2}\right\}^{1 / 2} .
$$

Under the assumptions $(\mathrm{Q})(1)-(\mathrm{Q})(3),(\mathrm{A} 1)$ and $(\mathrm{A} 2)$ will be verified in the second part of the paper. We turn now to the verification of (A3). For that purpose, introduce $H_{h}=\left\{\psi \in C(0, X) \mid \psi(0)=\psi(X)=0, \quad \psi\right.$ linear on $\left(x_{i-1}, x_{i}\right), \quad i=$ $1, \ldots, N\}$. Note that $H_{h}$ is isomorphic to $U_{h}$. Furthermore, if $\psi_{h}=\left\{\psi\left(x_{i}\right) \mid 1 \leqslant i \leqslant\right.$ $N-1\}$, then there exist two constants $c_{1}, c_{2}$ independent of $h$ such that

$$
c_{2}\left|\psi_{h}\right|_{h} \leqslant|\psi| \leqslant c_{1}\left|\psi_{h}\right|_{h}, \quad \forall \psi \in U_{h} .
$$

Also, for a function $f \in C(0, \infty)$ with $f(0)=0$, let $I_{h} f \in H_{h}$ be its interpolant satisfying $\left(I_{h} f\right)\left(x_{i}\right)=f\left(x_{i}\right), 1 \leqslant i \leqslant N-1$.

We now prove the following

LEMMA 1.4. Let $E_{k}$ be the invariant subspace corresponding to the eigenvalue $\lambda_{k}$, $1 \leqslant k$. Then under the assumptions $(\mathrm{Q})(1)-(\mathrm{Q})(2)$, there exists a sequence $\left\{\varepsilon_{h}\right\}$ such that $\lim _{h \rightarrow 0} \varepsilon_{h}=0$ and

$$
\left|f-I_{h} f\right| \leqslant \varepsilon_{h}|f|, \quad f \in E_{k} .
$$

Proof. We prove the assertion with $|f|=1$. Choose $h$ sufficiently small so that the interval $(0, X-h)$ includes the $k-1$ zeros of every eigenfunction; thus, on $(X-h, \infty), f(x)$, and consequently $I_{h} f$, keeps a constant sign.

We have the trivial inequality

$$
\left|f-I_{h} f\right|^{2} \leqslant \int_{0}^{X-h}\left[f(x)-\left(I_{h} f\right)(x)\right]^{2} d x+2 \int_{X-h}^{\infty}[f(x)]^{2} d x .
$$


As $f(x)$ decays exponentially to zero, $h$ is also chosen so that on $(X-h, \infty)$, $|f(x)| \leqslant c_{k} \exp \left(-d_{k} x\right), c_{k}$ and $d_{k}$ depending on $f$ and $E_{k}$ only. Since $f$ is $C^{\infty}$, it is well known that

$$
\left\{\int_{0}^{X-h}\left[f(x)-\left(I_{h} f\right)(x)\right]^{2} d x\right\}^{1 / 2} \leqslant \operatorname{ch}^{2}\left\{\int_{0}^{X-h}\left[f^{\prime \prime}(x)\right]^{2} d x\right\}^{1 / 2},
$$

with $c$ independent of $h$ and $X$. Furthermore, since

$$
-f^{\prime \prime}(x)+q(x) f(x)=\lambda_{k} f(x), \quad 0<x<\infty,
$$

one obtains, using $(\mathrm{Q})(2)$,

$$
\left\{\int_{0}^{X-h}\left|f(x)-\left(I_{h} f\right)(x)\right|^{2} d x\right\}^{1 / 2} \leqslant c h^{2}\left[\left|\lambda_{k}\right|+M\right] .
$$

Turning to the second term, $2 \int_{X-h}^{\infty}|f(x)|^{2} d x$, it is bounded by $\delta_{h}=$ $2\left(c_{k}^{2} / d_{k}\right) \exp \left\{-2 d_{k}(X-h)\right\}$. Letting $\varepsilon_{h}^{2}=\max \left\{\delta_{h}, c^{2} h^{4}\left(\left|\lambda_{k}\right|+M\right)^{2}\right\}$, we conclude the result.

If we write now $f_{h}=r_{h} f=\left\{f\left(x_{i}\right)\right\}, 1 \leqslant i \leqslant N-1$, we have the following

LEMMA 1.5. Let $E_{k}$ be the invariant subspace corresponding to the eigenvalue $\lambda_{k}$, $1 \leqslant k$. Let also $E_{k, h}=r_{h} E_{k}$, and $r_{h}^{E}: E_{k} \rightarrow E_{k, h}$. Then for $h$ small enough, $E_{k, h}$ and $r_{h}^{E}$ satisfy condition (A3).

Proof. From the identity $f_{h}=r_{h} f=r_{h} I_{h} f$, and (1.9), we have

$$
c_{2}\left|f_{h}\right|_{h} \leqslant\left|I_{h} f\right| \leqslant c_{1}\left|f_{h}\right|_{h} .
$$

Thus, for $|f|=1$, using Lemma 1.3, we may write

$$
c_{2}\left|f_{h}\right|_{h} \leqslant\left|I_{h} f\right| \leqslant|f|+\left|f-I_{h} f\right| \leqslant 1+\varepsilon_{h}
$$

thus giving $\left|r_{h}^{E}\right|_{U, U_{h}} \leqslant c_{1}$. As $h$ is chosen so that $(0, X)$ includes the $k-1$ zeros of every eigenfunction, one concludes that $r_{h}^{E}$ is bijective and $\operatorname{dim}\left(E_{k, h}\right)=1$.

Finally, consider $f_{h} \in U_{h}$ such that $\left|f_{h}\right|_{h}=1$, and $f \in E_{k}$ such that $f_{h}=r_{h}^{E} f$; from $|f| \leqslant\left|I_{h} f\right|+\left|f-I_{h} f\right| \leqslant\left|I_{h} f\right|+\varepsilon_{h}|f|$, one concludes for $h$ sufficiently small (as $\left.\varepsilon_{h} \rightarrow 0\right)$ that $\left(1-\varepsilon_{h}\right)|f| \leqslant\left|I_{h} f\right|$. Using again (1.10), one obtains

$$
\left|\left(r_{h}^{E}\right)^{-1}\right|_{U_{h}, U} \leqslant c_{2} \text {. }
$$

We turn finally to the estimation of the discretization error over the subspace $E_{k}$, $\gamma_{h}=\sup _{f \in E_{k}:|f|=1}\left|r_{h} L f-L_{h} r_{h} f\right|_{h}$. We have the following

LEMmA 1.6. Let $E_{k}$ be the invariant subspace corresponding to the eigenvalue $\lambda_{k}$, $k \geqslant 1$. Under the assumptions $(\mathrm{Q})(1)-(\mathrm{Q})(3)$, and assuming $q^{\prime}, q^{\prime \prime}, q^{\prime \prime \prime}, q^{(4)}$ are bounded on $(0, \infty)$, the following inequality is valid for $h$ sufficiently small:

$$
\gamma_{h} \leqslant 3 h^{4}\left(C_{1}+h^{-13 / 2}|f(X)|\right) .
$$


Proof. We take $f$ such that $|f|=1$. Let $\sigma_{h}=r_{h} L f-L_{h} r_{h} f \in R^{N-1}$. Let also $R_{h}$ be the linear transformation in $R^{N-1}$, represented by the tridiagonal matrix

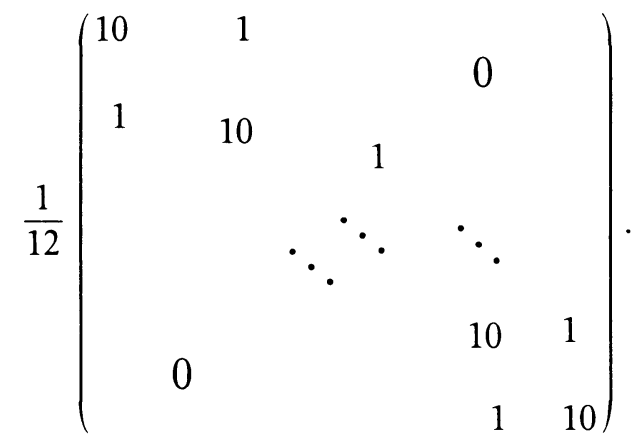

Note that

$$
\begin{aligned}
\left(R_{h} \sigma_{h}\right)_{i}= & \left(f_{i-1}^{\prime \prime}+10 f_{i}^{\prime \prime}+f_{i+1}^{\prime \prime}\right) / 12+\left(f_{i-1}-2 f_{i}+f_{i+1}\right) / h \\
& +\delta_{i, N-1}\left(f^{\prime \prime}(X) / 12-f(X) / h^{2}\right), \quad 1 \leqslant i \leqslant N-1,
\end{aligned}
$$

where $\delta_{i, k}$ is the Kronecker delta. The assumptions on $q$ permit the use of the Taylor expansion to obtain

$$
\begin{aligned}
\left(R_{h} \sigma_{h}\right)_{i}= & \frac{1}{120 h^{2}}\left[\int_{x_{i}}^{x_{i+1}}\left(x-x_{i}\right)^{5} f^{(6)}(x) d x+\int_{x_{i}}^{x_{i-1}}\left(x-x_{i}\right)^{5} f^{(6)}(x) d x\right] \\
& +\frac{1}{72}\left[\int_{x_{i}}^{x_{i+1}}\left(x-x_{i}\right)^{3} f^{(6)}(x) d x+\int_{x_{i}}^{x_{i-1}}\left(x-x_{i}\right)^{3} f^{(6)}(x) d x\right] \\
& +\delta_{i, N-1}\left(f^{\prime \prime}(X) / 12-f(X) / h^{2}\right)
\end{aligned}
$$

and

$$
\begin{aligned}
\left(R_{h} \sigma_{h}\right)_{i} \leqslant & e h^{4-1 / 2}\left[\left\{\int_{x_{i}}^{x_{i+1}}\left|f^{(6)}(x)\right|^{2} d x\right\}^{1 / 2}+\left\{\int_{x_{i-1}}^{x_{i}}\left|f^{(6)}(x)\right|^{2} d x\right\}^{1 / 2}\right] \\
& +\delta_{i, N-1}\left|f^{\prime \prime}(X) / 12-f(X) / h^{2}\right| \quad\left(e \cong 7.7 \times 10^{-3}\right)
\end{aligned}
$$

This implies

$$
\left[\sum_{i=1}^{N-1} h\left|\left(R_{h} \sigma_{h}\right)_{i}\right|^{2}\right]^{1 / 2} \leqslant 2 e h^{4}\left(\int_{0}^{X}\left|f^{(6)}(x)\right|^{2} d x\right)^{1 / 2}+\sqrt{h}\left|f^{\prime \prime}(X) / 12-f(X) / h^{2}\right|
$$

Consider now the equation $-f^{\prime \prime}(x)+q(x) f(x)=\lambda_{k} f(x)$; multiplication by $f$ and integration from 0 to $\infty$ yields

$$
\int_{0}^{\infty} f^{\prime 2}(x) d x+\int_{0}^{\infty} q(x) f^{2}(x) d x=\lambda_{k} \int_{0}^{\infty} f^{2}(x) d x,
$$

and therefore $\left|f^{\prime}\right|^{2} \leqslant M_{k}|f|^{2}$, where $M_{k}=\sup _{x \in(0, \infty)}\left|\lambda_{k}-q(x)\right|$. Successive differentiation of the above equation allows us to obtain $\left|f^{(j)}\right| \leqslant A_{j}|f|, 2 \leqslant j \leqslant 6$. Since $|f|=1$, one obtains for $h$ sufficiently small

$$
\begin{gathered}
\left\{\sum_{i=1}^{N-1} h\left|\left(R_{h} \sigma_{h}\right)_{i}\right|^{2}\right\}^{1 / 2} \leqslant 2 e h^{4} A_{6}+2 h^{-5 / 2}|f(X)|, \\
\left|R_{h} \sigma_{h}\right|_{h} \leqslant 2 h^{4}\left(e A_{6}+h^{-13 / 2}|f(X)|\right) .
\end{gathered}
$$

From $\left|R_{h} \sigma_{h}\right|_{h} \geqslant \frac{2}{3}\left|\sigma_{h}\right|_{h}$ one obtains the result. 
From the above, one clearly deduces

COROLlaRY 1.1. There exist choices for $h, X(h)$ such that for the system (1.1)-(1.3) Numerov's scheme yields a discretization error of order $O\left(h^{4}\right)$ over $E_{k}$, the invariant subspace corresponding to the eigenvalue $\lambda_{k}$.

Proof. Since $|f(x)| \leqslant c_{k} \exp \left(-d_{k} x\right)$ for $x$ sufficiently large, any choice for which $X(h)=O\left(h^{-m}\right), m>0$, will make $h^{-13 / 2}|f(X)|$ bounded as $h \rightarrow 0$. For example, $h=1 / n, X(h)=n, N(h)=n^{2}$, is a trivial choice. A more practical one for computer use is $h=1 / 2 m, X(h)=m^{i}, i>1, N(h)=2^{m} m^{i}$, which also yields the required result.

Remark. In the last choice, note that if one uses $i=1$, then $h^{-13 / 2}|f(X)| \rightarrow \infty$ as $h \rightarrow 0$.

We state finally a last theorem based on Theorems 1.1 and 1.2.

THEOREM 1.3. Under the assumptions of Lemma 1.6, for every isolated eigenvalue $\lambda_{k}, k \geqslant 1$, with multiplicity 1 of the operator $L$ in (1.1)-(1.3), the Numerov scheme yields a sequence of operators $L_{h}: R^{N-1} \rightarrow R^{N-1}$, and a sequence of isolated eigenvalues $\lambda_{k, h}$ of $L_{h}$, with the same multiplicity as $\lambda_{k}$, such that for some choices of $\{h, X(h)\}$ one has

$$
\left|\lambda_{k}-\lambda_{k, h}\right| \leqslant c h^{4}, \quad \hat{\delta}_{h}\left(r_{h} E_{k}, F_{k, h}\right) \leqslant c h^{4} .
$$

( $F_{k, h}$ in $R^{N-1}$ is the invariant subspace corresponding to $\lambda_{k, h}$ )

1.4. Application to Regular Sturm-Liouville Problems. Consider the eigenvalue problem

where

$$
\begin{gathered}
-\frac{d}{d x}\left[q(x) \frac{d y}{d x}\right]+s(x) y=\lambda p(x) y, \quad a<x<b, \\
y(a)=y(b)=0
\end{gathered}
$$

$$
p, q \text { and } s \text { are continuous and positive on }[a, b] .
$$

It is well known [9] that under the assumption (C1) there exists an increasing sequence of eigenvalues $\lambda_{1}<\lambda_{2}<\cdots<\lambda_{n}<\cdots$ that approach $\infty$, each having multiplicity 1 . The eigenfunction corresponding to $\lambda_{n}$ has exactly $n-1$ zeros in the open interval $(a, b)$.

Furthermore, we assume sufficient regularity on $p, q$ and $s$, so that $y \in C^{4}(a, b)$; for example,

$$
q \in C^{3}(a, b) ; \quad p, s \in C^{2}(a, b) .
$$

Consider a partition $x=a+i h, 0 \leqslant i \leqslant N$, with $N h=b-a$, and a discretization of (1.11) based on the central difference formula,

$$
\begin{gathered}
\delta_{h / 2} y(x)=(y(x+h / 2)-y(x-h / 2)) / h, \\
-\delta_{h / 2}\left(q_{i} \delta_{h / 2} Y_{i}\right)+s_{i} Y_{i}=\lambda_{h} p_{i} Y_{i}, \quad 0<i<N, \\
Y_{0}=Y_{N}=0 .
\end{gathered}
$$

To abide by our original notation, $L$ and $L_{h}$ are defined by

$$
\begin{gathered}
L y=\left(-\left(q(x) y^{\prime}\right)^{\prime}+s(x) y\right) / p(x), \\
\left(L_{h} Y\right)_{i}=\left(-\delta_{h / 2}\left(q_{i} \delta_{h / 2} Y_{i}\right)+s_{i} Y_{i}\right) / p_{i}, \quad 0<i<N,
\end{gathered}
$$

where $Y \in R^{N-1}$. 
As in Subsection 1.3, we let $U=L^{2}(a, b), U_{h}=R^{N-1}$, with respective norms

$$
|y|=\left\{\int_{a}^{b}[y(x)]^{2} d x\right\}^{1 / 2} \text { and }|Y|_{h}=\left\{\sum_{i=1}^{N-1} h Y_{i}^{2}\right\}^{1 / 2}, \quad y \in U, Y \in U_{h} .
$$

(A1) and (A2) will be verified in the second part of this paper. The verification of (A3) is similar to that of the Numerov scheme in Subsection 1.3, i.e., one introduces the subspace $H_{h}=\left\{\psi \in C(a, b) \mid \psi(a)=\psi(b)=0, \psi\right.$ linear on $\left(x_{i}, x_{i+1}\right), i=$ $0, \ldots, N-1\}$, and obtains analogues of Lemmas 1.4 and 1.5 in the newly introduced norms.

Let finally $\lambda_{k}$ be an isolated eigenvalue of $L$, with $E_{k}$ its invariant subspace, and $\gamma_{h}=\sup _{f \in E_{h}:|f|=1}\left|r_{h} L f-L_{h} r_{h} f\right|_{h}$. The estimation of $\gamma_{h}$ is based on the formula

$$
\begin{aligned}
\delta_{h / 2}\left(q_{i} \delta_{h / 2} y_{i}\right)= & \left(q(x) y^{\prime}(x)\right)^{\prime}+\frac{1}{2 h} \int_{x_{i-1 / 2}}^{x_{i+1 / 2}}\left(x-x_{i}\right)^{2}\left(q y^{\prime}\right)^{\prime \prime \prime} d x \\
& +\frac{q_{i+1 / 2}}{6 h^{2}} \int_{x_{i}}^{x_{i+1}}\left(x-x_{i+1 / 2}\right)^{3} y^{(4)}(x) d x \\
& +\frac{q_{i-1 / 2}}{6 h^{2}} \int_{x_{i-1}}^{x_{i}}\left(x-x_{i-1 / 2}\right)^{3} y^{(4)}(x) d x+\frac{h}{24} \int_{x_{i-1 / 2}}^{x_{i+1 / 2}}\left(q y^{\prime \prime \prime}\right)^{\prime} d x,
\end{aligned}
$$

which allows us to prove

LEMMA 1.7. Let $E_{k}$ be the invariant subspace corresponding to the eigenvalue $\lambda_{k}$, $k \geqslant 1$. Then under the assumption $(\mathrm{C} 2)$, the following inequality is valid:

$$
\left|r_{h} L f-L_{h} r_{h} f\right|_{h} \leqslant \frac{h^{2}}{\sqrt{80} \underline{p}}\left(\left|\left(q f^{\prime}\right)^{\prime \prime \prime}\right|^{2}+\bar{q}^{2}\left|f^{(4)}\right|^{2}+\left|\left(q f^{\prime \prime \prime}\right)^{\prime}\right|^{2}\right)^{1 / 2}, \quad f \in E_{k},
$$

with $p=\inf _{x} p(x)$ and $\bar{q}=\sup _{x}|q(x)|$.

Proof. We take $f \in E_{k}$ such that $|f|=1$. Let $\sigma_{h}=r_{h} L f-L_{h} r_{h} f \in R^{N-1}$. Note that

$$
\sigma_{h, i}=\left(-\left(q(x) f^{\prime}(x)\right)_{x=x_{i}}^{\prime}+\delta_{h / 2}\left(q_{i} \delta_{h / 2} f_{i}\right)\right) / p_{i}, \quad 0<i<N .
$$

Using (1.14), one bounds $\sigma_{h, i}$ as follows:

$$
\begin{aligned}
p_{i}\left|\sigma_{h, i}\right| \leqslant & \frac{1}{8 \sqrt{5}} h^{2-1 / 2}\left\{\int_{x_{i-1 / 2}}^{x_{i+1 / 2}}\left|\left(q f^{\prime}\right)^{\prime \prime \prime}\right|^{2} d x\right\}^{1 / 2} \\
& +\frac{q_{i+1 / 2}}{48 \sqrt{7}} h^{2-1 / 2}\left\{\int_{x_{i}}^{x_{i+1}}\left|f^{(4)}(x)\right|^{2} d x\right\}^{1 / 2} \\
& +\frac{q_{i-1 / 2} h^{2-1 / 2}}{48 \sqrt{7}} k\left\{\int_{x_{i-1}}^{x_{i}}\left|f^{(4)}(x)\right|^{2} d x\right\}^{1 / 2} \\
& +\frac{h^{2-1 / 2}}{24}\left\{\int_{x_{i-1 / 2}}^{x_{i+1 / 2}}\left|\left(q f^{\prime \prime \prime}\right)^{\prime}\right|^{2} d x\right\} .
\end{aligned}
$$


Thus, if $\bar{q}=\sup _{x \in(a, b)}|q(x)|$ and $\underline{p}=\inf _{x \in(a, b)} p(x)$,

$$
\begin{aligned}
p_{i}^{2} h\left|\sigma_{h, i}\right|^{2} \leqslant & \frac{1}{80} h^{4} \int_{x_{i-1 / 2}}^{x_{i+1 / 2}}\left|\left(q f^{\prime}\right)^{\prime \prime \prime}\right|^{2} d x+\frac{\bar{q}^{2} h^{4}}{4032} \int_{x_{i-1}}^{x_{i+1}}\left|f^{(4)}(x)\right|^{2} d x \\
& +\frac{h^{4}}{576} \int_{x_{i-1 / 2}}^{x_{i+1 / 2}}\left|\left(q f^{\prime \prime \prime}\right)^{\prime}\right|^{2} d x,
\end{aligned}
$$

and therefore

$$
\sum_{i=1}^{N-1} h\left|\sigma_{h, i}\right|^{2} \leqslant \frac{h^{4}}{\underline{p}^{2}}\left(\frac{1}{80}\left|\left(q f^{\prime}\right)^{\prime \prime \prime}\right|^{2}+\frac{\bar{q}^{2}}{2016}\left|f^{(4)}\right|^{2}+\frac{1}{576}\left|\left(q f^{\prime \prime \prime}\right)^{\prime}\right|^{2}\right),
$$

which proves our lemma.

To obtain final eigenvalue-eigenvector estimates, we note first that, when multiplying (1.11) by $y(x)$ and integrating by parts from $a$ to $b$,

$$
\int_{a}^{b} q(x) f^{\prime 2} d x+\int_{a}^{b} s(x) f^{2} d x=\lambda_{k} \int_{a}^{b} p(x) f^{2} d x, \quad f \in E_{k},
$$

whicn yieıus

$$
\underline{q}\left|f^{\prime}\right|^{2} \leqslant\left(\sup _{x \in(a, b)}\left|\lambda_{k} p-s\right|\right)|f|^{2}=M|f|^{2}
$$

where $q$ is $\inf _{x \in(a, b)}|q|$. Using again (1.11), one bounds $\left|\left(q f^{\prime}\right)^{\prime}\right|$ and $\left|f^{\prime \prime}\right|$ in terms of $|f|$; specifically,

$$
\begin{gathered}
\left|\left(q f^{\prime}\right)^{\prime}\right| \leqslant M|f| \quad(M \text { defined as in }(1.15)), \\
\left|f^{\prime \prime}\right| \leqslant \frac{1}{q}\left(M+\bar{q}^{\prime} \sqrt{\frac{M}{q}}\right)|f| \quad\left(\bar{q}^{\prime}=\sup _{x}\left|q^{\prime}(x)\right|\right) .
\end{gathered}
$$

Successive differentiation allows us to bound $f^{\prime \prime \prime}, f^{(4)}$, and $\left(q f^{\prime}\right)^{\prime \prime \prime}$ in terms of $|f|$, which, together with Lemma 1.7, gives $\gamma_{h} \leqslant c h^{2}$, and hence the following theorem.

THEOREM 1.4. Let $E_{k}$ be the invariant subspace associated with $\lambda_{k}$, an eigenvalue of the regular Sturm-Liouville operator defined in (1.11). Then under the assumption (C2), the three-point central difference formula (1.12)-(1.13) yields a sequence of eigenvalues $\lambda_{k, h}$ with corresponding eigenspaces $E_{k, h} \subset R^{N-1}$ such that for all $h$,

$$
\left|\lambda_{k}-\lambda_{k, h}\right| \leqslant c h^{2}, \quad \hat{\delta}_{h}\left(r_{h} E_{k}, F_{k, h}\right) \leqslant c h^{2} .
$$

\section{Part 2. A Sufficient Condition for Stability.}

2.1. Definition and Results. In this part we show that conditions (A1) and (A2) follow from an analysis of the discretization error of the difference formula over a suitable subspace. Specifically, consider the system (1.1)-(1.3), whose eigenvalues are approximated using a difference method such as the Numerov scheme, and define the sequence of operators $\left\{L_{X}\right\}_{h}$ by

$$
\begin{gathered}
L_{X} y=-y^{\prime \prime}+q(x) y, \quad 0<x<X, \\
c y^{\prime}(0)+d y(0)=0, \\
y(X)=0 .
\end{gathered}
$$

Note that (1.7) discretizes (1.1)-(1.3) as well as (2.1)-(2.3). 
In (1.2) we considered without loss of generality the case $c=0$. Let $H_{h}=\{\psi \in$ $C(0, X) \mid \psi$ linear in $\left.\left(x_{i}, x_{i+1}\right), 0 \leqslant i \leqslant N-1, \psi(0)=\psi(X)=0\right\}$. Let $\gamma>0$ be such that $(-\infty, 0) \in \zeta\left(L_{X}+\gamma\right)$, the resolvent set of $L_{X}+\gamma$; for every $\psi \in H_{h}$, let $z=A_{X} \psi \in C^{2}(0, X)$ be uniquely defined by

$$
\left(L_{X}+\gamma\right) z=\psi, \quad z(0)=z(X)=0 .
$$

As in Part 1, we introduce the notations

$$
\begin{gathered}
f \in L^{2}(0, \infty), \quad|f|=\left\{\int_{0}^{\infty}|f(x)|^{2} d x\right\}^{1 / 2}, \\
f \in H^{1}(0, \infty), \quad\|f\|=\left\{|f|^{2}+\left|f^{\prime}\right|^{2}\right\}^{1 / 2} .
\end{gathered}
$$

Also between $R^{N-1}$ and $H_{h}$ we consider the mappings $r_{h}: H_{h} \rightarrow R^{N-1}$ and $p_{h}$ : $R^{N-1} \rightarrow H_{h}$, where, if $\left\{w_{h, i}(x)\right\}_{i}$ is the usual "hat" functions basis in $H_{h}$, and $c \in R^{N-1}, \psi=p_{h} c=\sum_{i=1}^{N-1} c_{i} w_{h, i}(x)$, and $c=r_{h} \psi$.

We may therefore consider the discrete norms on $H_{h}$ or $R^{N-1}$,

$$
\begin{aligned}
& \psi \in H_{h}, \quad|\psi|_{h}=\left\{\sum_{i=1}^{N-1} h\left|\left(r_{h} \psi\right)_{i}\right|^{2}\right\}^{1 / 2}, \\
& \psi \in H_{h}, \quad\|\psi\|_{h}=\left\{|\psi|_{h}^{2}+\left|\psi^{\prime}\right|^{2}\right\}^{1 / 2} .
\end{aligned}
$$

$|\cdot|_{h}$ shall be used without distinction on $H_{h}$ and $R^{N-1}$. One proves the existence of two constants $c_{1}, c_{2}$ independent of $h$ such that

$$
c_{2}|\psi| \leqslant|\psi|_{h} \leqslant c_{1}|\psi|, \quad c_{2}\|\psi\| \leqslant\|\psi\|_{h} \leqslant c_{1}\|\psi\| \text {. }
$$

Furthermore, one has naturally $|\psi|_{h} \leqslant\|\psi\|_{h}, \forall \psi \in H_{h}$. Note that (1.7) as a general difference scheme can be easily transformed to a mapping in $H_{h}$, by considering the mapping $p_{h} L_{h} r_{h}: H_{h} \rightarrow H_{h}$.

Our main result is as follows.

THEOREM 2.1. Assume the difference operator $L_{h}$ is selected for the numerical approximation of the spectrum of $L$. If $L_{h}$ and $L$ satisfy the condition

$$
\lim _{h \rightarrow 0} \sup _{\substack{\psi \in H_{h} \\\|\psi\|=1 \\ z=A_{X} \psi}}\left|r_{h} L_{X} z-L_{h} r_{h} z\right|_{h}=0,
$$

then $L_{h}$ satisfies properties (A1), (A2).

To prove this theorem, we need to introduce additional notations. Let

$$
\begin{aligned}
{[\psi, \phi]_{h} } & =\sum_{i=1}^{N-1} h\left(r_{h} \psi\right)_{i}\left(r_{h} \bar{\phi}\right)_{i} \quad(\bar{\phi}: \text { complex conjugate }), \\
(\psi, \phi)_{h} & =\int_{0}^{x} \psi^{\prime}(x) \bar{\phi}^{\prime}(x) d x+[\psi, \phi]_{h}, \quad \psi, \phi \in H_{h} .
\end{aligned}
$$

One then defines the sesquilinear form $a_{h}$ on $H_{h} \times H_{h}$ by

$$
a_{h}(\psi, \phi)=\left[p_{h}\left(L_{h}+\gamma\right) \psi, \phi\right]_{h}
$$

and assumes the existence of constants $\gamma_{0}, \gamma_{1}>0$ independent of $h$ such that

$$
a_{h}(\psi, \psi) \geqslant \gamma_{0}\|\psi\|_{h}^{2}, \quad\left|a_{h}(\phi, \psi)\right| \leqslant \gamma_{1}\|\phi\|_{h}\|\psi\|_{h} .
$$


This allows the use of the Lax-Milgram theorem to define the sequence of operators $\left\{B_{h}\right\}_{h}: H_{h} \rightarrow H_{h}$ by the relation $a_{h}\left(B_{h} \psi, \phi\right)=[\psi, \phi]_{h}, \forall \psi, \phi \in H_{h}$. Note also that

$$
\left\|B_{h} \psi\right\|_{h} \leqslant c|\psi|_{h}, \quad \forall \psi \in H_{h}(c \text { independent of } h) \text {. }
$$

The analysis of the spectrum of $L_{h}: R^{N-1} \rightarrow R^{N-1}$ is equivalent to the analysis of the spectrum of $B_{h}: H_{h} \rightarrow H_{h}$. For condition (A1), this is straightforward; condition (A2) is obtained below using $\|\cdot\|_{h}$, but the following lemma shows that this implies (A2) in $\mid \cdot l_{h}$.

LEMMA 2.1. For $h$ sufficiently small, $z \neq-\gamma$ and $z \in \zeta\left(L_{h}\right)$ if and only if $z_{1}=$ $1 /(z+\gamma) \in \zeta\left(L_{h}\right)$. Furthermore, $\left\|\left(B_{h}-z_{1}\right) \psi\right\|_{h} \geqslant c_{0}\|\psi\|_{h}, \forall \psi \in H_{h}$ ( $c_{0}$ independent of $h)$ implies $\left|\left(L_{h}-z\right) f\right|_{h} \geqslant c|f|_{h}, \forall f \in R^{N-1}$ (c independent of $\left.h\right)$.

Proof. The first part of this lemma can easily be seen from the identity

$$
\left[\left(p_{h} L_{h} r_{h}-z_{1}\right) \psi, \phi\right]_{h}=(z+\gamma) a_{h}\left(\left(z_{1}-B_{h}\right) \psi, \phi\right), \quad \forall \phi, \psi \in H_{h} .
$$

As for the second part, note that for $f \in R^{N-1}, p_{h} f=\psi \in H_{h}$, one has

$$
\left(L_{h}-z\right) f_{h}=\sup _{\substack{\psi \in H_{h} \\|\psi|_{h}=1}}\left|\left[\left(p_{h} L_{h} r_{h}-z\right) \psi, \phi\right]_{h}\right| \geqslant \sup _{\substack{\phi \in H_{h} \\|\phi|_{h}=1}}\left|a_{h}\left(\left(B_{h}-z_{1}\right) \psi, \phi\right)\right| .
$$

Taking $\phi=\left(B_{h}-z_{1}\right) \psi /\left|\left(B_{h}-z_{1}\right) \psi\right|_{h}$, and using (2.5), we get

$$
\left|\left(L_{h}-z\right) f\right|_{h} \geqslant \gamma_{0}|z+\gamma|\left\|\left(B_{h}-z_{1}\right) \phi\right\|_{h}^{2} /\left|\left(B_{h}-z_{1}\right) \phi\right|_{h} \text {. }
$$

One then has

$$
\left|\left(L_{h}-z\right) f\right|_{h} \geqslant \gamma_{0}|z+\gamma|\left\|\left(B_{h}-z_{1}\right) \psi\right\|_{h} \geqslant \gamma_{0} c_{0}|z+\gamma|\|\phi\|_{h} .
$$

Using (2.4), one obtains the result.

The proof of Theorem 2.1 is based on a perturbation theory result.

Theorem 2.2. Let $A_{h}^{1}, A_{h}^{2}: H_{h} \rightarrow H_{h}$ be such that

$$
\lim _{h \rightarrow 0} \sup _{\substack{\psi \in H_{h} \\\|\psi\|=1}}\left\|\left(A_{h}^{1}-A_{h}^{2}\right) \psi\right\|=0 .
$$

Then $A_{h}^{1}$ satisfies (A1) and (A2) if and only if $A_{h}^{2}$ satisfies (A1) and (A2).

Proof. Assume $A_{h}^{1}$ satisfies (A1) and (A2).

(i) To show that $A_{h}^{2}$ satisfies (A2), assume there exists $\left\{h_{i}\right\}$ and, correspondingly, $\psi_{i} \in H_{h_{i}},\left\|\psi_{i}\right\|=1$ such that $\lim _{h_{i} \rightarrow 0}\left\|\left(A_{h_{i}}^{2}-\mu\right) \psi_{i}\right\|=0$. Note

$$
\left\|\left(A_{h_{i}}^{1}-\mu\right) \psi\right\| \leqslant\left\|\left(A_{h_{i}}^{1}-A_{h_{i}}^{2}\right) \psi\right\|+\left\|\left(A_{h_{i}}^{2}-\mu\right) \psi\right\| \text {. }
$$

Thus, $\lim _{h_{1} \rightarrow 0}\left\|\left(A_{h_{t}}^{1}-\mu\right) \psi\right\|=0$ and $\mu \notin \zeta\left(A_{h_{t}}^{1}\right)$, which contradicts (A2) for $A_{h}^{1}$.

(ii) For (A1), let $\lambda$ be an isolated eigenvalue of finite algebraic multiplicity $m$, and $\Delta$ a disc centered at $\lambda$, with boundary $\Gamma$ such that for all $h$ sufficiently small, $\Delta$ contains $m$ eigenvalues of $A_{h}^{1}$ (repeated according to their multiplicities) converging to $\lambda ; E_{h}^{1}=(2 \pi i)^{-1} \int_{\Gamma} R_{z}\left(A_{h}^{1}\right) d z$ is the spectral projector corresponding to $A_{h}^{1}$ and $E_{h}^{1}\left(H_{h}\right)$, its invariant subspace. Similarly, $E_{h}^{2}=(2 \pi i)^{-1} \int_{\Gamma} R_{z}\left(A_{h}^{2}\right) d z$, and one notes that

$$
\lim _{h \rightarrow 0} \sup _{\substack{\psi \in H_{h} \\\|\psi\|=1}}\left\|\left(E_{h}^{1}-E_{h}^{2}\right) \psi\right\|=0
$$


This can be seen from the identity

$$
E_{h}^{1}-E_{h}^{2}=(2 \pi i)^{-1} \int_{\Gamma} R_{z}\left(A_{h}^{1}\right)\left(A_{h}^{2}-A_{h}^{1}\right) R_{z}\left(A_{h}^{2}\right) d z
$$

Using the first part of the theorem and (P), one obtains (2.7). Consider now the mapping $\bar{E}_{h}^{2}=\left.E_{h}^{2}\right|_{E_{h}^{1}\left(H_{h}\right)}: E_{h}^{1}\left(H_{h}\right) \rightarrow E_{h}^{2}\left(H_{h}\right)$. For $\psi \in H_{h}$ such that $\|\psi\|=1$, one has from (2.7) that $\lim _{h \rightarrow 0}\left\|\psi-\bar{E}_{h}^{2} \psi\right\|=0$, which obviously shows that for $h$ sufficiently small, $\bar{E}_{h}^{2}$ is injective. Hence $\operatorname{dim}\left(E_{h}^{1}\left(H_{h}\right)\right) \leqslant \operatorname{dim}\left(E_{h}^{2}\left(H_{h}\right)\right)$. A similar argument on $\bar{E}_{h}^{1}=\left.E_{h}^{1}\right|_{E_{h}^{2}\left(H_{h}\right)}: E_{h}^{2}\left(H_{h}\right) \rightarrow E_{h}^{1}\left(H_{h}\right)$ shows that $\operatorname{dim}\left(E_{h}^{2}\left(H_{h}\right)\right) \leqslant$ $\operatorname{dim}\left(E_{h}^{1}\left(H_{h}\right)\right)$, and therefore $\operatorname{dim}\left(E_{h}^{1}\left(H_{h}\right)\right)=\operatorname{dim}\left(E_{h}^{2}\left(H_{h}\right)\right)$.

2.2. Proof of Theorem 2.1. To obtain Theorem 2.1, we now relate the "difference operator" $B_{h}$ to the interpolation operator $C_{h}=\left.I_{h} A_{X}\right|_{H_{h}}$. This is done in

LEMMA 2.2. The difference approximation $B_{h}$ and the "interpolatory" approximation $\left.I_{h} A_{X}\right|_{H_{h}}$ satisfy the inequality

$$
\left\|\left(B_{h}-I_{h} A_{X}\right) \psi\right\| \leqslant c\left|r_{h} L_{X} z-L_{h} r_{h} z\right|_{h} \quad(c \text { independent of } h) .
$$

Proof. To obtain the result, let $e_{h}=B_{h} \psi-I_{h} A_{X} \psi$. With $z=A_{X} \psi$, consider $\sigma_{h}=r_{h} L_{X} z-L_{h} r_{h} z$, the discretization error associated with $z$, which can be written as $r_{h}\left(L_{X}-2 \alpha\right) z-\left(L_{h}-2 \alpha\right) r_{h} z$. Clearly, from $\sigma_{h} \in R^{N-1}$ one writes

$$
\left[p_{h} \sigma_{h}, \phi\right]_{h}=\left[p_{h} r_{h}\left(L_{X}-2 \alpha\right) z, \phi\right]_{h}-\left[p_{h}\left(L_{h}-2 \alpha\right) r_{h} z, \phi\right]_{h},
$$

and therefore, using $z=A_{X} \psi$ and the definition of $B_{h}$,

$$
[\psi, \phi]_{h}-a_{h}\left(p_{h} r_{h} z, \phi\right)=\left[p_{h} \sigma_{h}, \phi\right]_{h}, \quad a_{h}\left(B_{h} \psi-I_{h} A_{X} \psi, \phi\right)=\left[p_{h} \sigma_{h}, \phi\right]_{h} .
$$

Letting $\phi=e_{h}$ and using inequalities (2.4), one obtains the result.

Hence, if the discretization error is such that

$$
\lim _{h \rightarrow 0} \sup _{\substack{\psi \in H_{h} \\\|\psi\|=1 \\ z=A_{X} \psi}}\left|r_{h} L_{X} z-L_{h} r_{h} z\right|_{h}=0
$$

then $B_{h}$ and $C_{h}$ satisfy property (P), demonstrating clearly that Theorem 2.1 is a consequence of Theorem 2.2 and Lemma 2.2. The analysis of the convergence of $\sigma\left(L_{h}\right)$ depends on a well-known result of approximation theory.

Lemma 2.3. Assume q satisfies $(\mathrm{Q})(2)$ ( see Part 1$)$; then

$$
\left\|A_{X} \psi-I_{h} A_{X} \psi\right\|_{X} \leqslant c h|\psi| \text {. }
$$

Here $c$ is a constant independent of $h$ and $X .\left(\|\cdot\|_{X}\right.$ here is the $H^{1}(0, X)$-norm.)

Proof. One knows that $\left\|A_{X} \psi-I_{h} A_{X} \psi\right\|_{X}$ is bounded by

$$
\operatorname{ch}\left[\int_{0}^{X}\left|z^{\prime \prime}(x)\right|^{2} d x\right]^{1 / 2}, \quad \text { with } c \text { independent of } h \text { and } X \text {. }
$$

Furthermore, using trivial energy inequalities directly related to the equation $-z^{\prime \prime}+$ $q(x) z=\psi(x)$, one finds

$$
\left\{\int_{0}^{X}\left|z^{\prime \prime}(x)\right|^{2} d x\right\}^{1 / 2} \leqslant c(M, \alpha)\left\{\int_{0}^{X}|\psi|^{2} d x\right\}^{1 / 2},
$$

which yields the result. 
2.3. Corollaries for Bounded and Unbounded Domains. For bounded domains $X=a$, note that $A_{X}=A ; L_{X}=L . \sigma(A)$ includes only isolated eigenvalues of finite algebraic multiplicity. Furthermore, $A$ is compact in $L^{2}(0, a)$. In this case, Lemma 2.3 yields

$$
\left\|\left(A-C_{h}\right) \psi\right\| \leqslant \operatorname{ch}\|\psi\|, \quad \psi \in H_{h} .
$$

Moreover, $H_{h}$ is dense in $H^{1}(0, a)$; together with (2.8), this is sufficient, according to Descloux, Nassif and Rappaz [4], to have $\sigma\left(C_{h}\right)$ satisfy (A1) and (A2). This immediately yields

COROllary 2.1. Assume a difference operator $L_{h}$ is chosen to compute the eigenvalues of a differential operator $L$ defined by $L y=-y^{\prime \prime}+q(x) y, 0<x<a, y(0)=$ $y(a)=0$; then a sufficient condition for $\sigma\left(L_{h}\right)$ to satisfy properties (A1) and (A2) is that

$$
\lim _{h \rightarrow 0} \sup _{\substack{\psi \in H_{h} \\\|\psi\|=1 \\ z=A \psi}}\left|r_{h} L z-L_{h} r_{h} z\right|_{h}=0 .
$$

Furthermore, property (A1) is satisfied for every isolated eigenvalue of $L$.

For unbounded domains, our findings are based on Galerkin finite element approximation. Define first $\pi: H^{1}(0, \infty) \rightarrow H_{h}$, the $a$-projector, by the relation

$$
a(f, \psi)=a\left(\pi_{h} f, \psi\right), \quad \forall \psi \in H_{h} .
$$

Then

$$
A_{h}=\left.\pi_{h} A\right|_{H_{h}}: H_{h} \rightarrow H_{h}
$$

is the Galerkin approximation.

The following lemma is fundamental.

LeMma 2.4. The Galerkin approximation $A_{h}=\left.\pi_{h} A\right|_{H_{h}}$ and the interpolatory approximation $C_{h}=\left.I_{h} A_{X}\right|_{H_{h}}$ satisfy property $(\mathrm{P})$.

Proof. From the relation

$$
a\left(\left(A_{h}-C_{h}\right) \psi, \phi\right)=a_{h}\left(A_{X} \psi-I_{h} A_{X} \psi, \phi\right), \quad \forall \psi, \phi \in H_{h},
$$

one obtains

$$
\left\|\left(A_{h}-C_{h}\right) \psi\right\| \leqslant c\left\|A_{X} \psi-I_{h} A_{X} \psi\right\|_{X}
$$

Lemma 2.3 yields our results.

As a consequence one obtains

COROllaRy 2.2. Consider the difference operator $L_{h}$ used to compute the eigenvalues of the operator $L$ defined by

$$
L y=-y^{\prime \prime}+q(x) y, \quad 0<x<\infty, y(0)=0, y \text { bounded } .
$$

If $L_{h}$ is chosen such that

$$
\lim _{h \rightarrow 0} \sup _{\substack{\psi \in H_{h} \\\|\psi\|=1 \\ z=A_{X} \psi}}\left|r_{h} L_{X} z-L_{h} r_{h} z\right|_{h}=0,
$$


then $L_{h}$ satisfies (A1) and (A2) whenever the Galerkin approximation $G_{h}=A_{h}^{-1}-\gamma$ satisfies (A1) and (A2).

This corollary enables us to use previous results obtained earlier for Galerkin approximations. In particular, by use of the Courant principle [2] and the notion of essential numerical range, Descloux [3] has clearly demonstrated that one obtains (A1) and (A2) for Galerkin approximations outside the essential numerical range $\xi$. In the particular case where $q$ satisfies $(\mathrm{Q})(1)-(\mathrm{Q})(3), \xi$ is exactly the interval $[0,1 / \gamma]$, which forms the continuous spectrum.

Outside such an interval, $A$ has only isolated eigenvalues that can be approximated by the Galerkin method, and therefore by the difference operator $L_{h}$ satisfying property $(\mathrm{N})$.

2.4. Verification of (N) for Numerov's Scheme. Using the notations of Lemma 1.6, we write $\sigma_{h}=r_{h} L_{X} z-L_{h} r_{h} z$ for $z=A_{X} \psi, \psi \in U_{h}$.

We consider also the linear transformation in $R^{N-1}, R_{h}$, represented by the tridiagonal matrix

$$
\frac{1}{12}\left(\begin{array}{rrrrr}
10 & 1 & & & \\
1 & 10 & \ddots & \\
& & \ddots & \ddots & 1 \\
& & & & 1 \\
& 0 & & & 10
\end{array}\right) .
$$

Note that

$$
\begin{aligned}
\left(R_{h} \sigma_{h}\right)_{i}= & -\left(z_{i-1}^{\prime \prime}+10 z_{i}^{\prime \prime}+z_{i+1}^{\prime \prime}\right) / 12 \\
& +\left(z_{i-1}-2 z_{i}+z_{i+1}\right) / h^{2}, \quad 1 \leqslant i \leqslant N-1 .
\end{aligned}
$$

$\left(R_{h} \sigma_{h}\right)_{i}$ can be found to have the following expression:

$$
\begin{aligned}
\left(R_{h} \sigma_{h}\right)_{i}= & \frac{1}{2 h^{2}}\left[\int_{x_{i}}^{x_{i+1}}\left(x-x_{i}\right)^{2} z^{\prime \prime \prime}(x) d x-\int_{x_{i-1}}^{x_{i}}\left(x-x_{i}\right)^{2} z^{\prime \prime \prime}(x) d x\right] \\
& +\frac{1}{12}\left[\int_{x_{i}}^{x_{i+1}} z^{\prime \prime \prime}(x) d x-\int_{x_{i-1}}^{x_{i}} z^{\prime \prime \prime}(x) d x\right] .
\end{aligned}
$$

This leads to the following

LeMmA 2.5. Assuming $z^{\prime \prime \prime} \in L^{2}(0, X)$, one has

$$
\left|R_{h} \sigma_{h}\right|_{h} \leqslant \alpha_{0} h\left\{\int_{0}^{X}\left|z^{\prime \prime \prime}\right|^{2} d t\right\}^{1 / 2} \quad \text { with } \alpha_{0}=\frac{1}{6}+\frac{4}{\sqrt{5}}
$$

Proof. Use standard arguments based on energy inequalities. 
On the basis of Lemma 2.5 one obtains

LEMMA 2.6. Under the assumptions $(\mathrm{Q})(1)-(\mathrm{Q})(3)$ one has

$$
\lim _{h \rightarrow 0} \sup _{\substack{\psi \in H_{h} \\ z=A_{X} \psi \\\|\psi\|=1}}\left|r_{h} L_{X} z-L_{h} r_{h} z\right|_{h}=0 .
$$

Proof. Since $-z^{\prime \prime}+q(x) z=\psi, 0<x<X$, differentiation yields $z^{\prime \prime \prime}=q^{\prime} z+q z^{\prime}$ $-\psi^{\prime}$. Hence,

$$
\begin{aligned}
\left\{\int_{0}^{X}\left|z^{\prime \prime \prime}\right|^{2} d x\right\}^{1 / 2} \leqslant & \left\{\int_{0}^{X}\left(q^{\prime} z\right)^{2} d x\right\}^{1 / 2}+\left\{\int_{0}^{X}\left(q z^{\prime}\right)^{2} d x\right\}^{1 / 2} \\
& +\left\{\int_{0}^{X} \psi^{\prime 2} d x\right\}^{1 / 2} .
\end{aligned}
$$

From $(\mathrm{Q})(1)-(\mathrm{Q})(3)$ one concludes

$$
\left|z^{\prime \prime \prime}\right|_{L^{2}(0, X)} \leqslant l|z|_{L^{2}(0, X)}+M\left|z^{\prime}\right|_{L^{2}(0, X)}+\left|\psi^{\prime}\right|_{L^{2}(0, X)} .
$$

Using standard energy inequalities, one may bound $|z|_{L^{2}(0, X)}$ and $\left|z^{\prime}\right|_{L^{2}(0, X)}$ in terms of $|\psi|_{L^{2}(0, X)}$ which leads to

$$
\left\{\int_{0}^{X}\left|z^{\prime \prime \prime}\right|^{2} d x\right\}^{1 / 2} \leqslant c\|\psi\| .
$$

Lemma 2.5 completes the proof, as all the positive eigenvalues of $R_{h}$ are bounded independently of $h$.

This proves

THEOREM 2.2. Under the assumptions (Q)(1)-(Q)(3), the Numerov scheme satisfies condition (A1) for every isolated eigenvalue of finite multiplicity, and (A2) for every compact set of the resolvent set $\zeta(L)$.

2.5. Verification of (N) for the Central Difference Operator for the Regular Sturm-Liouville Operator. For the purpose on hand, we apply Corollary 2.1 to the difference scheme (1.12)-(1.13) for the approximate solution of (1.11). To complete our notations, let

$$
\begin{aligned}
& f \in L^{2}(a, b), \quad|f|=\left\{\int_{0}^{\infty}|f(x)|^{2} d x\right\}^{1 / 2}, \\
& f \in H^{1}(a, b), \quad\|f\|=\left\{|f|^{2}+\left|f^{\prime}\right|^{2}\right\}^{1 / 2}, \\
& f_{h} \in R^{N-1}, \quad\left|f_{h}\right|_{h}=\left\{\sum_{i=1}^{N-1} h\left|f_{h_{i}}\right|^{2}\right\}^{1 / 2} .
\end{aligned}
$$

Moreover, the definitions of Subsection 2.1 with respect to $H_{h}$ and $R^{N-1}$ are maintained, and therefore relations (2.4) and (2.5) remain valid. To analyze the discretization error over the set of solutions $z(x)$ of

$$
\begin{gathered}
-\left(q(x) z^{\prime}\right)^{\prime}+s(x) z=p(x) \psi(x), \quad a<x<b, \\
z(a)=z(b)=0,
\end{gathered}
$$


where $\psi \in H_{h}$, we use the following argument. Assuming $p, s \in H^{1}(a, b), q \in$ $H^{2}(a, b)$ and therefore $z \in H^{3}(a, b), q z^{\prime} \in H^{2}(a, b)$, one writes

$$
\begin{aligned}
\delta_{h / 2}\left(q_{i} \delta_{h / 2} z_{i}\right)= & \left(q(x) z^{\prime}\right)_{x=x_{i}}+\frac{1}{h} \int_{x_{i-1 / 2}}^{x_{i+1 / 2}}\left(x-x_{i}\right)\left(q(x) z^{\prime}\right)^{\prime \prime} d x \\
& +\frac{1}{2 h^{2}}\left[q_{i+1 / 2} \int_{x_{i}}^{x_{i+1}}\left(x-x_{i+1 / 2}\right)^{2} z^{\prime \prime \prime} d x\right. \\
& \left.-q_{i-1 / 2} \int_{x_{i-1}}^{x_{i}}\left(x-x_{i-1 / 2}\right)^{2} z^{\prime \prime \prime} d x\right] .
\end{aligned}
$$

If

$$
\sigma_{h}=r_{h} L z-L_{h} r_{h} z
$$

then

$$
\sigma_{h, i}=-\left(q(x) z^{\prime}\right)_{x=x_{i}}+\delta_{h / 2}\left(q_{i} \delta_{h / 2} z_{i}\right), \quad 0<i<N .
$$

Using (2.10), one obtains by standard techniques the following lemma.

Lemma 2.7. Assuming $z \in H^{3}(a, b), q z^{\prime} \in H^{2}(a, b)$, the discretization error of the difference scheme (1.12)-(1.13) for (2.9) satisfies

$$
\left|r_{h} L z-L_{h} r_{h} z\right|_{h} \leqslant \frac{h}{2 \underline{p}} \max \{1, \bar{q}\}\left\{2\left|z^{\prime \prime \prime}\right|^{2}+\left|\left(q z^{\prime}\right)^{\prime \prime}\right|^{2}\right\}^{1 / 2} .
$$

Again, using energy inequalities, one bounds the right-hand side of Lemma 2.7 in terms of $\|\psi\|$, which leads to

THEOREM 2.3. Under the assumptions of Lemma 2.7, the central difference scheme for the regular Sturm-Liouville problem satisfies condition (A1) for each eigenvalue, and (A2) for every compact set of the resolvent set.

2.6. Condition (N) for a 2-dimensional case. Consider the problem of finding the eigenvalues of the differential operator $L$ defined for a function $u(x, y)$ by

$$
\begin{gathered}
L u=-\Delta u=-\frac{\partial^{2} u}{\partial x^{2}}-\frac{\partial^{2} u}{\partial y^{2}}, \quad(x, y) \in D, \\
u(x, y)=0, \quad(x, y) \in \partial D,
\end{gathered}
$$

where

$$
D=\{(x, y) \mid 0<x<a, 0<y<b\} .
$$

Define the spaces $L^{2}(D)$ with norm $|\cdot|$ and inner product $[\cdot, \cdot]$, and $H^{1}(D)$ with norm $\|\cdot\|$ and inner product $(\cdot, \cdot)$. Let

$$
H_{0}^{1}(D)=\left\{\phi \in H^{1}(D) \mid \phi=0 \text { on } \partial D\right\} .
$$

With $h=\left(h_{1}, h_{2}\right) \in(0,1) \times(0,1)$, define the discrete domain

$$
D_{h}=\left\{\left(x_{i}, y_{i}\right) \mid x_{i}=i h_{1}, y_{j}=j h_{2}, 0<i<M, 0<j<N, i, j \text { integers }\right\} \text {. }
$$

For $u_{h} \equiv\left\{u_{h, i, j}\right\}_{0 \leqslant i \leqslant M, 0 \leqslant j \leqslant N}$ define the five-point difference operator $-\Delta_{h}$ given for $u_{h}: D_{h} \rightarrow R$ by

$$
\begin{gathered}
\left(-\Delta_{h} u_{h}\right)_{i, j}=\left(2 u_{i, j}-u_{i-1, j}-u_{i+1, j}\right) / h_{1}^{2} \\
+\left(2 u_{i, j}-u_{i, j-1}-u_{i, j+1}\right) / h_{2}^{2}, \quad i, j \in D_{h}, \\
u_{h, i, j}=0, \quad i, j \in \partial D_{h}
\end{gathered}
$$


(for simplicity we have written $u_{h, i, j}=u_{i, j}$ ). (2.13) and (2.14) can be reduced to finding the eigenvalues of the operator $L_{h}: R^{M-1} \times R^{N-1} \rightarrow R^{M-1} \times R^{N-1}$.

Let also

$$
\begin{aligned}
H_{h}= & \left\{\psi_{h} \in H^{1}(D) \cap C(D) \mid \psi_{h}=0 \text { on } \partial D,\right. \\
& \left.\psi_{h}(x, y)=a+b x+c y+d x y, x, y \in D_{i, j}, 0 \leqslant i<M, 0 \leqslant j<N\right\},
\end{aligned}
$$

with $D_{i, j}=\left\{(x, y) \in D \mid x_{i} \leqslant x \leqslant x_{i+1}, y_{j} \leqslant y \leqslant y_{j+1}\right\} . H_{h}$ is the well-known piecewise bilinear space.

Note that $H_{h} \subset H_{0}^{1}(D)$; furthermore, $\psi \in H_{h}, \psi_{x y} \in L^{2}(D)$. On $H_{h}$ consider the discrete norm $|\cdot|_{h}$ defined by

$$
|\psi|_{h}=\left\{\sum_{i=1}^{M-1} \sum_{j=1}^{N-1} h_{1} h_{2}\left|\psi_{i, j}\right|^{2}\right\}^{1 / 2},
$$

induced by the discrete inner product

$$
[\psi, \phi]_{h}=\sum_{i=1}^{M-1} \sum_{j=1}^{N-1} h_{1} h_{2} \psi_{i, j} \phi_{i, j},
$$

where

$$
\begin{gathered}
\psi_{i, j} \equiv \psi_{h}\left(x_{i}, y_{j}\right), \quad \phi_{i, j} \equiv \phi_{h}\left(x_{i}, y_{j}\right) ; \\
r_{h}: H^{1}(D) \cap C(D) \rightarrow R^{M-1} \times R^{N-1} \text { is defined by } \\
\quad\left(r_{h} f\right)_{i, j}=f\left(x_{i}, y_{j}\right), 0<i<M, 0<j<N .
\end{gathered}
$$

Note that $|\cdot|_{h}$ can be considered as a norm on $R^{M-1} \times R^{N-1}$. Let $\sigma_{h}$ be the discretization error, defined for $z \in C^{2}(D)$ by

$$
\sigma_{h}(z)=r_{h} L z-L_{h} r_{h} z
$$

Our basic result is that $L_{h}$ defined in (2.13), (2.14) satisfies condition (N). This will imply the stability results (A1)-(A2) for the 5-point difference scheme used to approximate the frequencies of vibration of the operator $-\Delta$. This is summarized in

THEOREM 2.4. We have

$$
\lim _{h \rightarrow 0} \sup _{\substack{\psi \in H_{h} \\\|\psi\|=1 \\ z=A \psi}}\left|r_{h} L z-L_{h} r_{h} z\right|_{h}=0 .
$$

Several preliminary results are needed, which we simply summarize to avoid technical details.

LEMMA 2.8. There exists a constant $c$ independent of $h$ such that

$$
\left|\sigma_{h}\right|_{h}^{2} \leqslant c\left(h_{1}^{2}\left|z_{x x x}\right|^{2}+h_{2}^{2}\left|z_{y y y}\right|^{2}+h_{1}^{2} h_{2}\left|z_{x x x y}\right|^{2}+h_{2}^{2} h_{1}\left|z_{y y y x}\right|^{2}\right) .
$$

If $z$ is the solution of $-\Delta z=\psi \in H_{h}, z=0$ on $\partial D$, then using Fourier analysis one proves that

$$
\begin{array}{cl}
\left|z_{x x x}\right|^{2} \leqslant\left|\psi_{x}\right|^{2}, & \left|z_{y y y}\right|^{2} \leqslant\left|\psi_{y}\right|^{2}, \\
\left|z_{x x x y}\right|^{2} \leqslant\left|\psi_{x y}\right|^{2}, & \left|z_{y y y x}\right|^{2} \leqslant\left|\psi_{x y}\right|^{2},
\end{array}
$$


and by standard calculation on the subspace $H_{h}$ one gets

$$
\left|\psi_{x y}\right| \leqslant \frac{c}{h_{1}}\left|\psi_{y}\right|, \quad c \text { independent of } h .
$$

Combining Lemma 2.8 with (2.15) and (2.16), one obtains

LEMMA 2.9. There exists a constant $c$ independent of $h$ such that

$$
\left|r_{h} L z-L_{h} r_{h} z\right|_{h}^{2} \leqslant c\left(h_{1}^{2}+h_{2}^{2}+h_{1}+h_{2}\right)\|\psi\|^{2},
$$

where

$$
-\Delta z=\psi \in H_{h}, \quad z=0 \quad \text { on } \partial D .
$$

Consequently, one obtains Theorem 2.4.

Department of Mathematics

American University of Beirut

Beirut. Lebanon

1. J. H. Bramble \& J. E. Osborn, "Rate of convergence estimates for nonselfadjoint eigenvalue approximations," Math. Comp., v. 27, 1973, pp. 525-549.

2. F. Chatelin, Théorie de l'Approximation des Opérateurs Linéaires, Application au Calcul des Valeurs Propres d'Opérateurs Différentiels et Intégraux, Lecture Notes, Grenoble University, 1977.

3. J. Descloux, "Essential numerical range of an operator with respect to a coercive form and the approximation of its spectrum by the Galerkin method," SIAM J. Numer. Anal., v. 18, 1981, pp. $1128-1133$.

4. J. Descloux, N. R. Nassif \& J. Rappaz, “On spectral approximation, Part 1: The problem of convergence; Part 2: Error estimates for the Galerkin method," RAIRO Anal. Numér., v. 12, 1978, pp. 97-112; pp. 113-119.

5. J. Descloux, N. R. Nassif \& J. Rappaz, “On properties of spectral approximations," in Equadiff $I V$, Lecture Notes in Math., vol. 703, Springer-Verlag, Berlin and New York, 1977, pp. 81-85.

6. R. D. GrigoriefF, "Diskrete Approximation von Eigenwertproblemen, Part 1: Qualitative Konvergenz; Part 2: Konvergenzordnung; Part 3: Asymptotische Entwicklungen," Numer. Math., v. 24, 1975, pp. 355-374; pp. 415-453; v. 25, 1975, pp. 79-97.

7. H. O. KreISS, "Difference approximations for boundary and eigenvalue problems for ordinary differential equations," Math. Comp., v. 26, 1972, pp. 605-624.

8. B. V. Numerov, Monthly Notices Roy. Astronom. Soc., v. 84, (180), 1924, p. 592.

9. E. C. Titchmarsh, Eigenfunction Expansions, Oxford Univ. Press, London, 1962.

10. J. E. OsBorn, "Spectral approximations for compact operators," Math. Comp., v. 29, 1975, pp. $712-725$.

11. G. Strang \& G. J. Fix, An Analysis of the Finite Element Method, Prentice-Hall, Englewood Cliffs, N. J., 1973.

12. F. Stummel, "Elliptische Differenzenoperatoren unter Dirichlet Randbedingungen," Math. Z., v. 97, 1967, pp. 169-211.

13. G. M. VAinikKo, "A difference method for ordinary differential equations," U.S.S.R. Comput. Math. and Math. Phvs., v. 9, 1969, pp. 1-32.

14. J. H. Wilkinson, The Algebraic Eigenvalue Problem, Oxford Univ. Press, London, 1965. 